\title{
Solving the Direct and Inverse Geodetic Problems on the Ellipsoid by Numerical Integration
}

\author{
Lars E. Sjöberg ${ }^{1}$ and Masoud Shirazian²
}

\begin{abstract}
Taking advantage of numerical integration, we solve the direct and inverse geodetic problems on the ellipsoid. In general, the solutions are composed of a strict solution for the sphere plus a correction to the ellipsoid determined by numerical integration. Primarily the solutions are integrals along the geodesic with respect to the reduced latitude or azimuth, but these techniques either have problems when the integral passes a vertex (i.e., point with maximum/minimum latitude of the arc) or a singularity at the equator. These problems are eliminated when using Bessel's idea of integration along the geocentric angle of the great circle of an auxiliary sphere. Hence, this is the preferred method. The solutions are validated by some numerical comparisons to Vincenty's iterative formulas, showing agreements to within $2 \times 10^{-10}$ of geodesic length (or $3.1 \mathrm{~mm}$ ) and $4 \times 10^{-5}$ as seconds of azimuth and position for baselines in the range of 19,000 km. DOI: 10.1061/(ASCE)SU.1943-5428.0000061. () 2012 American Society of Civil Engineers.
\end{abstract}

CE Database subject headings: Geodetic surveys; Numerical analysis.

Author keywords: Direct problem; Inverse problem; Geodesic.

\section{Introduction}

Since the times of Clairaut (1713-1765) and the work of Bessel (1826), the direct and inverse geodetic problems on the ellipsoid have attracted the attention of numerous geodesists. Although ellipsoidal solutions are of less importance in the space age, problems related with geodesics may still be relevant. Examples can be found, e.g., in geographic information systems in the application of the United Nations Convention of the Law of the Sea, military surveys, and in the optimization of aircraft's and ship's routes. Also, one may still claim that a two-dimensional (2D) network on the ellipsoid has some advantages to those of a modern three-dimensional (3D) network.

The classical solutions of these problems were frequently in the forms of power series of the ellipsoidal eccentricity. This technique also refers to solving the area of a closed geodesic polygon, as discussed in Sjöberg (2006). Today, Vincenty's (1975) approximate solutions based on the series given in Rainsford (1955) seem to be the preferred formulas for solving the direct and inverse problems of the geodesic. On the other hand, considering the capability of modern computers, it is natural to take advantage of methods employing numerical integration. As one of the pioneering researchers to such methods, Kivioja (1971) solved the direct problem by simple numerical integrations of two of the basic differential equations of the geodesic. Heck (1987), Klotz (1991), and Schmidt (1999) presented some contributions along this line, but they still relied on series expansions. Thomas and Featherstone (2005) improved Kivioja's technique and claimed that there is a remarkable

\footnotetext{
${ }^{1}$ Royal Institute of Technology, Division of Geodesy and Geoinformatics, SE100 44 Stockholm, Sweden (corresponding author). E-mail: 1sjo@kth.se

${ }^{2}$ Royal Institute of Technology, Division of Geodesy and Geoinformatics, SE-100 44 Stockholm, Sweden. E-mail: masoudsh@kth.se

Note. This manuscript was submitted on November 30, 2010; approved on June 8, 2011; published online on January 17, 2012. Discussion period open until July 1, 2012; separate discussions must be submitted for individual papers. This paper is part of the Journal of Surveying Engineering, Vol. 138, No. 1, February 1, 2012. CASCE, ISSN 0733-9453/2012/1-9$16 / \$ 25.00$.
}

numerical agreement between this method and that of Vincenty for baselines ranging to as much as $18,000 \mathrm{~km}$. Nevertheless, as Kivioja's method solves only the direct problem, it could not be used to check Vincenty's inverse formulas.

On the contrary, Sjöberg (2006a) solved the direct and inverse problems by decomposing the solutions to those on a sphere and their corrections to the ellipsoid. The spherical solutions are given in closed forms, while the corrections to the ellipsoid are elliptical integrals of magnitudes of order of the first eccentricity square $\left(e^{2}\right)$, suitable for numerical integration. Although some improvements of this technique were achieved through the discussion between Schmidt (2006) and Sjöberg (2006b), the method still did not perform well for long geodesics extending to and beyond the maximum or minimum latitudes (the vertices) of the geodesic. Converting the previous integrals along reduced latitudes to integrals along azimuths is used here as one tool to solve this problem. In addition, a modified Bessel technique, with integration along the geocentric angle, will be used. Part of the inverse problem is also to solve for Clairaut's constant, which was treated by Sjöberg (2007).

\section{Basic Formulas}

Let us assume that the semimajor and semiminor axes $a$ and $b$ of an ellipsoid are defined. From these we may compute the first eccentricity by $e=\sqrt{a^{2}-b^{2}} / a$. Then the generating differential equations of the geodesic (line) on the ellipsoid can be written as (e.g., Heck 1987; Klotz 1991; Schmidt 1999)

$$
d s=a \sqrt{\frac{1-e^{2} \cos ^{2} \beta}{\cos ^{2} \beta-c^{2}}} \cos \beta d \beta
$$

and

$$
d \lambda=c \sqrt{\frac{1-e^{2} \cos ^{2} \beta}{\cos ^{2} \beta-c^{2}}} \frac{d \beta}{\cos \beta}
$$

Here $d s$ and $d \lambda$ are the infinitesimal arc-length and longitude of the geodesic, and $\beta$ is the reduced latitude. Furthermore 


$$
c=\cos \beta \sin \alpha
$$

is called Clairaut's constant, where $\alpha$ is the azimuth. By integrating Eqs. (1) and (2) between the points with latitudes $\beta_{1}$ and $\beta_{2}>\beta_{1}$, one obtains

$$
s=a \int_{\beta_{1}}^{\beta_{2}} \sqrt{\frac{1-e^{2} \cos ^{2} \beta}{\cos ^{2} \beta-c^{2}}} \cos \beta d \beta
$$

and

$$
\lambda_{2}=\lambda_{1}+c \int_{\beta_{1}}^{\beta_{2}} \sqrt{\frac{1-e^{2} \cos ^{2} \beta}{\cos ^{2} \beta-c^{2}}} \frac{d \beta}{\cos \beta}
$$

These integrals are not useful for the second terminal point located at a vertex of the geodesic or beyond this point. In this case one may change the integration variable from latitude to azimuth. If we exclude the azimuth $\alpha=0$ or $\pi$, implying a geodesic directed along a meridian, one obtains from Eq. (3) that

$$
\cos \beta=c / \sin \alpha \quad \text { and } \quad \sin \beta= \pm \sqrt{1-c^{2} / \sin ^{2} \alpha}
$$

and by differentiating Eq. (3) and inserting Eqs. (6) one obtains also

$$
d \beta= \pm \cot \beta \cot \alpha d \alpha= \pm \frac{c \cos \alpha d \alpha}{\sin \alpha \sqrt{\sin ^{2} \alpha-c^{2}}}
$$

Furthermore, by inserting Eqs. (6) and (7) into Eqs. (1) and (2) and integrating from $\alpha_{1}$ to $\alpha_{2}$, we arrive at

$$
s= \pm a c \int_{\alpha_{1}}^{\alpha_{2}} \sqrt{\frac{\sin ^{2} \alpha-e^{2} c^{2}}{\sin ^{2} \alpha-c^{2}}} \frac{d \alpha}{\sin ^{2} \alpha}
$$

and

$$
\lambda_{2}=\lambda_{1} \pm \int_{\alpha_{1}}^{\alpha_{2}} \sqrt{\frac{\sin ^{2} \alpha-e^{2} c^{2}}{\sin ^{2} \alpha-c^{2}}} d \alpha
$$

where the sign convention is so that $s$ is always positive. (Below we will also consider only $\lambda_{1} \leq \lambda_{2}$.)

Finally, we provide the following useful transformations for some trigonometric formulas between the geographical and reduced latitudes ( $\varphi$ and $\beta$, respectively):

$$
\begin{aligned}
\tan \beta & =\sqrt{1-e^{2}} \tan \varphi, \\
\sin \beta & =\sqrt{1-e^{2}} \sin \varphi / \sqrt{1-e^{2} \sin ^{2} \varphi} ; \\
\cos \beta & =\cos \varphi / \sqrt{1-e^{2} \sin ^{2} \varphi} \xi
\end{aligned}
$$

\section{Solutions of the Integral Formulas}

Eqs. (4), (5), (8), and (9) are elliptic integrals, which cannot be strictly integrated and have nonclosed form solutions. The standard solution for such an integral in ellipsoidal geodesy is to develop it into a series of the eccentricity. However, having access to modern computers, our strategy is different: each integral is decomposed to its solution on the sphere, and a small correction (of the relative order of $e^{2}$ ) is added to obtain the strict integral on the ellipsoid. The spherical part has a simple and closed form solution, while the correction is to be determined by numerical integration. In the solutions, we will always assume that the two terminal points of the geodesic are arranged such that $\lambda_{1} \leq \lambda_{2}$, which implies that $0 \leq c \leq$ 1 (or $0 \leq \alpha \leq \pi)$.

Because Eqs. (4) and (5) have singular integrands when the geodesic passes a vertex, Eqs. (8) and (9) are preferred there. Unfortunately, Eqs. (8) and (9) yield singular integrands at the equator, suggesting that Eqs. (4) and (5) are used in the vicinity of this point on the geodesic. These problems are considered in the solutions that follow for arc length and longitude difference.

- Solution for the arc-length.

Three cases must be distinguished: solutions along the meridian, solutions along the equator, and other solutions.

- Solutions along the meridian.

In this case, the azimuth of the geodesic and the Clairaut constant are both zero.

Case (a) $\left(\lambda_{1}=\lambda_{2}\right)$ : From Eq. (1) it follows that

$$
s=a \int_{\beta_{1}}^{\beta_{2}} \sqrt{1-e^{2} \cos ^{2} \beta} d \beta=a\left(\beta_{2}-\beta_{1}\right)+d s
$$

where

$$
\begin{aligned}
d s & =a \int_{\beta_{1}}^{\beta_{2}}\left(\sqrt{1-e^{2} \cos ^{2} \beta}-1\right) d \beta \\
& =-a e^{2} \int_{\beta_{1}}^{\beta_{2}} \frac{\cos ^{2} \beta d \beta}{\sqrt{1-e^{2} \cos ^{2} \beta}+1} \\
& =-a e^{2} \int_{x_{1}=\sin \beta_{1}}^{x_{2}=\sin \beta_{2}} \frac{\sqrt{1-x^{2}}}{1+\sqrt{1-e^{2}\left(1-x^{2}\right)}} d x
\end{aligned}
$$

Case (b) $\left(\lambda_{2}=\lambda_{1}+\pi\right)$ : The terminal points are located on opposite sides of the pole, and the integral of Eq. (11a) can be written

$$
s=a \sum_{i=1}^{2}\left|\int_{\beta_{i}}^{\pi / 2} \sqrt{1-e^{2} \cos ^{2} \beta} d \beta\right|=S+d s
$$

where

$$
S=a \sum_{i=1}^{2} \int_{\beta_{i}}^{\pi / 2} d \beta=a\left(\pi-\beta_{1}-\beta_{2}\right)
$$

and

$$
d s=-e^{2} a \sum_{i=1}^{2}\left|\int_{x_{i}}^{1} \frac{\sqrt{1-x^{2}} d x}{1+\sqrt{1-e^{2}\left(1-x^{2}\right)}}\right|
$$

where $x_{i}=\sin \beta_{i}$.

- Solution on the equator.

If the Clairaut constant $c=1$, the geodesic is on the equator and $s=a\left(\lambda_{2}-\lambda_{1}\right)$, where $\lambda_{i}$ are the longitudes of the terminal points with $\lambda_{2}>\lambda_{1}$.

- Solutions for $0<c<1$.

If $0<c<1$, two cases should be considered.

Case (a) $\left(\alpha_{1}<\alpha_{2} \leq \pi / 2\right.$ or $\left.\pi / 2 \leq \alpha_{2}<\alpha_{1}\right)$ : This case implies that the geodesic between the terminal points does not contain the vertex point $P_{0}$, i.e., the point with maximum/ minimum latitude $\left(\beta_{0}\right)$ and azimuth $\pi / 2$ of the geodesic. Then Eq. (8) can be decomposed into

$$
s=S+d s
$$


where

$$
S=a c \int_{\alpha_{1}}^{\alpha_{2}} \frac{d \alpha}{\sin \alpha \sqrt{\sin ^{2} \alpha-c^{2}}}= \pm a c \int_{x_{1}=\sin \alpha_{1}}^{x_{2}=\sin \alpha_{2}} \frac{d x}{x \sqrt{1-x^{2}} \sqrt{x^{2}-c^{2}}}= \pm a \arcsin \left(\frac{1}{x} \sqrt{\frac{x^{2}-c^{2}}{1-c^{2}}}\right)_{x_{1}}^{x_{2}}
$$

where the sign is chosen such that $S$ becomes positive.

Furthermore, if the geodesic does not pass the equator, then

$$
d s= \pm e^{2} a c^{3} \int_{y_{1}=\cos \alpha_{1}}^{y_{2}=\cos \alpha_{2}} \frac{d y}{\left(1-y^{2}\right)^{3 / 2} \sqrt{1-c^{2}-y^{2}}\left(\sqrt{1-y^{2}}+\sqrt{1-y^{2}-e^{2} c^{2}}\right)}
$$

and if the geodesic passes the equator, then

$$
d s=-a e^{2} \int_{x_{1}=\sin \beta_{1}}^{x_{2}=\sin \beta_{2}} \frac{d x}{\sqrt{1-c^{2}-x^{2}}\left(1+\sqrt{1-e^{2}+e^{2} x^{2}}\right)}
$$

If one of the terminal points equals $P_{0}$ [i.e., the point of the geodesic with azimuth $\left.( \pm \pi / 2)\right], d s$ can be computed by a combination of Eqs. $(13 b)$ and $(13 d)$.

Case (b) $\left(\alpha_{1}<\pi / 2<\alpha_{2}\right)$ : In this case the integral passes a vertex, and one can obtain from Eq. (8) that

$$
s=S+d s
$$

where $S$ is again given by the first part of Eq. (13b), but the latter part must change sign (as $\cos \alpha_{i}$ becomes negative for $\alpha_{i}>\pi / 2$ ) to contribute positively to the arc-length, i.e.,

$$
S=a c \int_{\alpha_{1}}^{\alpha_{2}} \frac{d \alpha}{\sin \alpha \sqrt{\sin ^{2} \alpha-c^{2}}}=+a c \sum_{i=1}^{2} \int_{x_{1}=\sin \alpha_{i}}^{1} \frac{d x}{x \sqrt{1-x^{2}} \sqrt{x^{2}-c^{2}}}=a \sum_{i=1}^{2} \arcsin \left(\frac{1}{x} \sqrt{\frac{x^{2}-c^{2}}{1-c^{2}}}\right)_{x=x_{i}}^{x=1}
$$

and (if the geodesic does not pass the equator between the terminal points)

$$
d s=-a e^{2} c^{3} \int_{\alpha_{1}}^{\alpha_{2}} \frac{d \alpha}{\sin ^{2} \alpha \sqrt{\sin ^{2} \alpha-c^{2}}\left(\sqrt{\sin ^{2} \alpha-e^{2} c^{2}}+\sin \alpha\right)}=a e^{2} c^{3} \int_{y_{1}=\cos \alpha_{1}}^{y_{2}=\cos \alpha_{2}} \frac{d y}{\left(1-y^{2}\right)^{3 / 2} \sqrt{1-c^{2}-y^{2}}\left(\sqrt{1-e^{2} c^{2}}+\sqrt{1-y^{2}}\right)}
$$

If the geodesic passes the equator between the terminal points, $d s$ can be decomposed as

$$
d s=d s_{1}+d s_{2}
$$

where

$$
d s_{1}=-a e^{2} c^{3} \int_{\beta_{1}}^{\beta_{3}} \frac{\cos \beta d \beta}{\sqrt{\cos ^{2} \beta-c^{2}}\left(1+\sqrt{1-e^{2} \cos ^{2} \beta}\right)}
$$

and

$$
d s_{2}=a e^{2} c^{3} \int_{\cos \alpha_{3}}^{\cos \alpha_{2}} \frac{d y}{\left(1-y^{2}\right)^{3 / 2} \sqrt{1-c^{2}-y^{2}}\left(\sqrt{1-e^{2} c^{2}}+\sqrt{1-y^{2}}\right)}
$$

Here $\beta_{3}$ is the latitude of an arbitrary point on the geodesic (with $\beta_{3}<\beta_{0}$ ) on the opposite side of the equator with respect to $P_{1}$, and $\alpha_{3}=\pi-\arcsin \left(c / \cos \beta_{3}\right)$.

- Solution for the longitude difference.

- The geodesic along the meridian.

If $c=0$, then the longitude difference is zero.

- The geodesic along the equator.

If $c=1$, then the longitude difference is given previously as $s / a$.

- General case with $0<c<1$.

If $0<c<1$, it follows from Eq. (9) that

$$
\lambda_{2}-\lambda_{1}=\Lambda+d \lambda
$$

where

$$
\Lambda=\int_{\alpha_{1}}^{\alpha_{2}} \frac{\sin \alpha d \alpha}{\sqrt{\sin ^{2} \alpha-c^{2}}}
$$

and

$$
d \lambda=-e^{2} c^{2} \int_{\alpha_{1}}^{\alpha_{2}} \frac{d \alpha}{\sqrt{\sin ^{2} \alpha-c^{2}}\left(\sin \alpha+\sqrt{\sin ^{2} \alpha-e^{2} c^{2}}\right)}
$$

To practically solve Eqs. (16b) and (16c) some special cases must be considered.

Case (a). If $\alpha_{1}<\alpha_{2} \leq \pi / 2$ or $\pi / 2 \leq \alpha_{1}<\alpha_{2}$, and the geodesic does not pass the equator between the terminal points, then 
Eqs. (16b) and (16c) can be written as

$$
\Lambda= \pm \int_{y_{2}=\cos \alpha_{2}}^{y_{1}=\cos \alpha_{1}} \frac{d y}{\sqrt{1-c^{2}-y^{2}}}= \pm \arcsin \left(\frac{y_{i}}{\sqrt{1-c^{2}}}\right)_{i=1}^{i=2}
$$

and

$$
d \lambda=\mp e^{2} c^{2} \int_{y_{2}}^{y_{1}} \frac{d y}{\sqrt{1-c^{2}-y^{2}}\left(\sqrt{1-y^{2}}+\sqrt{1-y^{2}-e^{2} c^{2}}\right)}
$$

In Eqs. (17a) and (17b) the upper and lower signs apply for $\alpha_{1}<\alpha_{2} \leq \pi / 2$ and $\pi / 2 \leq \alpha_{1}<\alpha_{2}$, respectively.

Case (b). If $\alpha_{1}<\pi / 2<\alpha_{2}$ and the geodesic does not pass the equator between the terminal points, then Eqs. (16b) and (16c) can be written as

$$
\Lambda=\sum_{i=1}^{2} \int_{0}^{\cos \alpha_{i}} \frac{d y}{\sqrt{1-c^{2}-y_{i}^{2}}}=\sum_{i=1}^{2} \arcsin \left(\frac{\cos \alpha_{i}}{\sqrt{1-c^{2}}}\right)
$$

and

$$
\begin{aligned}
d \lambda= & -e^{2} c^{2} \sum_{i=1}^{2} \\
& \times \int_{0}^{\cos \alpha_{i}} \frac{d y}{\sqrt{1-y^{2}} \sqrt{1-y^{2}-c^{2}}\left(\sqrt{1-y^{2}}+\sqrt{1-y^{2}-e^{2} c^{2}}\right)}
\end{aligned}
$$

Alternatively, a modified Eq. (16c) can be used for computing $d \lambda$.

Case (c). If the geodesic passes the equator between the terminal points, Eqs. (17b) and (18b) will be singular at the equator. To avoid this problem, the computation for $d \lambda$ must be decomposed into three components: two similar to Eq. (18b), and one integral with respect to latitude for a section passing the equator.

The above solutions will be used to solve the direct and indirect geodetic problems on the ellipsoid.

\section{Direct Problem on the Ellipsoid}

Given: Let the coordinates of latitude $\left(\varphi_{1}\right)$ and longitude $\left(\lambda_{1}\right)$ of point $P_{1}$ be given. Also given are the azimuth $\alpha_{1}$ at $P_{1}$ and the geodesic arc-length $s$ to another point $P_{2}$.

Problem: Determine the coordinates of $P_{2}$. (See Fig. 1.)

Solution: First convert the given geodetic latitude to reduced latitudes by Eq. (10).

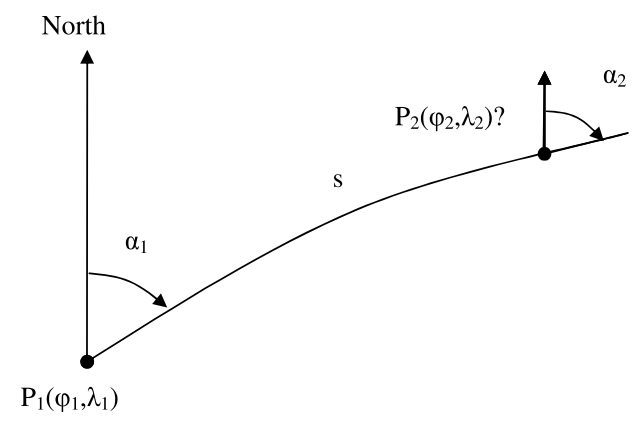

Fig. 1. The direct problem on the ellipsoid
Case $1\left(\alpha_{1}=0\right)$. In this case $\lambda_{2}=\lambda_{1}$, Clairaut's constant is zero, and the latitude of $P_{2}$ is given by

$$
\beta_{2}=\beta_{1}+(s-d s) / a
$$

where $d s$ and $\beta_{2}$ are determined by iteratively applying Eqs. (11b) and (19).

Case $2\left(\varphi_{1}=0\right.$ and $\left.\alpha_{1}=90^{\circ}\right)$. Here the geodesic runs along the equator, implying that $\beta_{2}=0$ and $\lambda_{2}=\lambda_{1}+s / a$.

Case 3 (all situations except those in Cases 1 and 2).

The problem can be solved as follows.

- Compute Clairaut's constant using the following equation for the geodesic by Eq. (3):

$$
c=\cos \beta_{1} \sin \alpha_{1}
$$

- Compute the latitude of $P_{2}$.

- First compute the geodesic arc $s_{0}$ from $P_{1}$ to $P_{0}$ (i.e., the point with maximum latitude) according to Eqs. (13a)-(13d).

- If $s=s_{0}, \quad$ it follows that $\beta_{2}=\arccos (c)$

- If $s \neq s_{0}$, one obtains from Eqs. (13a) and (13b) with $x_{1}=$ $\sin \alpha_{1}$ that

$$
\frac{1}{x_{2}} \sqrt{\frac{x_{2}^{2}-c^{2}}{1-c^{2}}}=\sin \left[\frac{s-d s}{a}+\arcsin \left(\frac{1}{x_{1}} \sqrt{\frac{x_{1}^{2}-c^{2}}{1-c^{2}}}\right)\right]=A
$$

which yields

$$
\begin{aligned}
x_{2}= & \sin \alpha_{2}=\frac{c}{\sqrt{1-A^{2}\left(1-c^{2}\right)}} \quad \text { and } \\
\cos \beta_{2} & =\sqrt{1-A^{2}(1-c)^{2}}
\end{aligned}
$$

Here $d s$ and $A$ are determined by iterating Eqs. (13c), (22a), and $(22 b)$.

The procedure of computing $\lambda_{2}$ is explained in Eqs. (16a)-(16c).

\section{Inverse Problem}

The coordinates $\left(\varphi_{i}, \lambda_{i}\right) ; i=1,2$ of points $P_{1}$ and $P_{2}$ are given.

Problem: Determine the length of the geodesic between $P_{1}$ and $P_{2}$ and its azimuths at the end points.

Solution: First, convert the geodetic latitudes to reduced latitudes.

Case $1\left(\lambda_{1}=\lambda_{2}\right)$. In this case the geodesic runs along a meridian, implying that $\alpha_{1}=\alpha_{2}=0$ or $\pi$. The arc-length of the geodesic follows from Eqs. (11a) and (11b).

Case $2\left(\beta_{1}=\beta_{2}=0\right.$ and $|\pi-\Delta \lambda|>h$, where $\Delta \lambda=\lambda_{2}-\lambda_{1}$ and $h \approx \pi e^{2} / 2 \approx 36^{\prime} 09^{\prime \prime}$. In this case $\alpha_{1}=\alpha_{2}= \pm \pi / 2$, implying that the geodesic is located along the equator (see Schmidt 2006 and Sjöberg 2006a), and its length becomes

$$
s=a \Delta \lambda
$$

Case 3. If $\beta_{1}=\beta_{2}=0$ and $|\pi-\Delta \lambda| \leq h$, then Clairaut's constant follows from (see Sjöberg 2006b)

$$
c=\frac{\pi-\Delta \lambda}{2 e^{2} I} \approx \frac{2}{e^{2}}\left(1-\frac{\Delta \lambda}{\pi}\right)
$$

where $I \approx \pi / 4$ is a definite integral given in Sjöberg (2006b). 
The azimuths at the terminal points are now given by Eq. (3) as $\pm \arcsin c$, and the arc-length follows from Eqs. (14a), (14b), and $(15 a)-(15 c)$.

Case 4. If $\lambda_{1} \neq \lambda_{2}$ and $\beta_{1} \neq 0$ and/or $\beta_{2} \neq 0$, Clairaut's constant is given as follows (cf. Sjöberg 2006a):

$$
c=1 / \sqrt{1+t_{0}^{2}}
$$

where

$$
t_{0}^{2}=\frac{t_{1}^{2}+t_{2}^{2}-2 t_{1} t_{2} \cos D L}{\sin ^{2} D L}, \quad \text { with } \quad t_{i}=\tan \beta_{i}
$$

and

$$
D L=\lambda_{2}-\lambda_{1}-d \lambda \neq \pi
$$

Here $d \lambda$ is the same as in Eqs. (16a) and (16c).

Case 5. If $D L=\pi$, Eq. (25b) is not valid, and in this case $c$ follows from Eq. (24).

1. The azimuths at the end points of the geodesic follow from Eq. (3) as

$$
\alpha_{i}=\arcsin \left(c / \cos \beta_{i}\right), \quad i=1,2
$$

or

$$
\pi-\alpha_{i}=\arcsin \left(c / \cos \beta_{i}\right), \quad i=1,2
$$

or a mixture of Eqs. (26a) and (26b), dependent on the location of the vertex versus the given points;

2. The arc-length follows from Eqs. $(13 a)-(13 d)$ and (15a)-(15c).

\section{Modified Bessel Method}

Bessel (1826) took advantage of an auxiliary sphere for the integration along the geodesic. Such a technique will also be used here, but while Bessel presented the solutions as power series expressions in the eccentricity of the ellipsoid, we follow our previous strategy to divide the solutions into closed expressions for the sphere and small corrections, suitable for numerical integrations.

1. Solution for the arc-length.

Consider the integration of Eq. (1) for a small arc not including the vertex:

$$
s=a \int_{\beta_{1}}^{\beta} \sqrt{\frac{1-e^{2} \cos ^{2} \beta}{\cos ^{2} \beta-c^{2}}} \cos \beta d \beta=S+d s
$$

where

$$
S=a\left(\arcsin \frac{\sin \beta}{\sqrt{1-c^{2}}}-\arcsin \frac{\sin \beta_{1}}{\sqrt{1-c^{2}}}\right)=a\left(\gamma-\gamma_{1}\right)
$$

and

$$
d s=a \int_{\beta_{1}}^{\beta} \frac{\sqrt{1-e^{2} \cos ^{2} \beta}-1}{\sqrt{\cos ^{2} \beta-c^{2}}} \cos \beta d \beta
$$

Because the last integral will not be valid when the geodesic passes a vertex, we will now transform it to an integral with respect to the geocentric angle $\psi$ (not to be mixed with geocentric latitude). To do so we introduce

$$
S=a \psi
$$

and by considering Eq. (27b) one therefore obtains

$$
\sin \psi=\sin \left(\gamma-\gamma_{1}\right), \quad \cos \psi=\cos \left(\gamma-\gamma_{1}\right)
$$

and

$$
\gamma=\gamma(\psi)=\gamma_{1}+\psi
$$

By differentiating the first equation for a constant $\gamma_{1}$ one obtains also

$$
d \psi=\frac{\cos \left(\gamma-\gamma_{1}\right)}{\cos \psi} d \gamma=d \gamma
$$

Moreover, from the definition of $\gamma$ in Eq. (27b) one can write

$$
\sin \gamma=\frac{\sin \beta}{\sqrt{1-c^{2}}}
$$

and its differential becomes

$$
d(\sin \gamma)=\cos \gamma d \gamma=d\left(\frac{\sin \beta}{\sqrt{1-c^{2}}}\right)=\frac{\cos \beta}{\sqrt{1-c^{2}}} d \beta
$$

yielding

$$
d \psi=d \gamma=\frac{\cos \beta}{\sqrt{\cos ^{2} \beta-c^{2}}} d \beta
$$

This implies that Eq. (27a) can be written as

$$
s=a \psi+a \int_{0}^{\psi}\left(\sqrt{1-e^{2} \cos ^{2} \beta}-1\right) d \psi
$$

It remains to express the square root as a function of $\psi$. To achieve this, we present the following proposition and corollary. (The proof of the proposition was given above. The proof of the corollary follows directly from the proposition.)

Proposition 1: $\sin \beta=\sqrt{1-c^{2}} \sin \gamma$, where $\gamma=\gamma(\psi)=$ $\gamma_{1}+\psi$

Corollary 1: $\sqrt{1-e^{2} \cos ^{2} \beta}=\sqrt{1-e^{2}} \sqrt{1+k^{2} \sin ^{2} \gamma}$, where $k^{2}=\left(a^{2}-b^{2}\right) / b^{2}\left(1-c^{2}\right)$.

Using the corollary, we thus arrive at the following solution for the geodesic arc-length:

$$
\begin{aligned}
& s=b\left[\psi+k^{2} \int_{0}^{\psi} \frac{\sin ^{2} \gamma(\psi)}{1+\sqrt{1+k^{2} \sin ^{2} \gamma}(\psi)} d \psi\right], \\
& \text { where } b=a \sqrt{1-e^{2}}
\end{aligned}
$$

or

$$
\begin{aligned}
s= & b\left\{\left[1+\frac{k^{2}}{4}\right] \psi+\frac{k^{2}}{4} \frac{\sin 2 \gamma_{1}-\sin 2 \gamma(\psi)}{2}\right. \\
& \left.-\frac{k^{4}}{2} \int_{\gamma_{1}}^{\psi+\gamma_{1}} \frac{\sin ^{4} \gamma(\psi)}{\left[1+\sqrt{1+k^{2} \sin ^{2} \gamma(\psi)}\right]^{2}} d \psi\right\}
\end{aligned}
$$

In Eqs. (34a) and (34b), the integrals can be determined by numerical integration.

Alternatively, considering that the length of any geodesic from one point to its antipode is of the order of $a \pi \approx 20,000 \mathrm{~km}$, it follows that $a \pi e^{4}<0.9 \mathrm{~km}, a \pi e^{6}<6 \mathrm{~m}$, and $a \pi e^{8}<4 \mathrm{~cm}$. Hence, using the series expansions

$$
1+\sqrt{1+x^{2}} \approx 2\left(1+\frac{x^{2}}{4}-\frac{x^{4}}{16}\right)
$$

and 


$$
\left(1+\sqrt{1+x^{2}}\right)^{-2} \approx\left[2\left(1+x^{2} / 4-x^{4} / 16\right)\right]^{-2} \approx \frac{1}{4}-\frac{x^{2}}{8}+\frac{5 x^{4}}{64}
$$

and by inserting the latter series with $x=k \sin \gamma$ into the integral of Eq. (34b), one arrives at

$$
\begin{aligned}
s \approx & b\left\{\left[1+\frac{k^{2}}{4}\right] \psi+\frac{k^{2}}{4} \frac{\sin 2 \gamma_{1}-\sin 2 \gamma(\psi)}{2}\right. \\
& \left.-\frac{k^{4}}{2} I S^{4}+\frac{k^{6}}{16} I S^{6}-\frac{5 k^{8}}{32} I S^{8}\right\}
\end{aligned}
$$

where

$$
\begin{aligned}
I S^{2 n}= & \int_{\gamma_{1}}^{\gamma} \sin ^{2 n} \gamma d \gamma=-\left\{\frac { \operatorname { c o s } x } { 2 n } \left[\sin ^{2 n-1} x+\frac{2 n-1}{2 n-2} \sin ^{2 n-3} x\right.\right. \\
& +\frac{(2 n-1)(2 n-3)}{(2 n-2)(2 n-4)} \sin ^{2 n-5} x+\ldots \\
& \left.\left.+\frac{(2 n-1) \ldots 3 \cdot 1}{(2 n-2) \ldots 4 \cdot 2} \sin x\right]\right\}_{x=\gamma}^{\gamma_{1}}={ }_{2} F_{1}\left(\frac{1}{2}, \frac{1}{2}-n, \frac{3}{2}, \cos ^{2} \gamma\right) \\
& -{ }_{2} F_{1}\left(\frac{1}{2}, \frac{1}{2}-n, \frac{3}{2}, \cos ^{2} \gamma_{1}\right)
\end{aligned}
$$

Here ${ }_{2} F_{1}$ is Gauss' hypergeometric function.

2. Solution for the longitude difference.

The integral of Eq. (2) becomes the following for $\beta_{1}<\beta$ :

$$
\Delta \lambda=c \int_{\beta_{1}}^{\beta} \sqrt{\frac{1-e^{2} \cos ^{2} \beta}{\cos ^{2} \beta-c^{2}}} \frac{d \beta}{\cos \beta}=\Lambda+d \lambda
$$

where

$$
\begin{aligned}
\Lambda & =c \int_{\beta_{1}}^{\beta} \frac{d \beta}{\sqrt{\cos ^{2} \beta-c^{2}} \cos \beta} \\
& =\arctan \left(\frac{c \sin \beta}{\sqrt{\cos ^{2} \beta-c^{2}}}\right)-\arctan \left(\frac{c \sin \beta_{1}}{\sqrt{\cos ^{2} \beta_{1}-c^{2}}}\right)
\end{aligned}
$$

or, after applying Proposition 1 and Corollary 1

$$
\Lambda=\arctan (c \tan \gamma)-\arctan \left(c \tan \gamma_{1}\right)
$$

and

$$
\begin{aligned}
d \lambda & =c \int_{\beta_{1}}^{\beta} \frac{\sqrt{1-e^{2} \cos ^{2} \beta}-1}{\cos \beta \sqrt{\cos ^{2} \beta-c^{2}}} d \beta \\
& =c \int_{0}^{\psi} \frac{\sqrt{1-e^{2} \cos ^{2} \beta}-1}{\cos ^{2} \beta} d \psi \\
& =-c e^{2} \int_{0}^{\psi} \frac{d \psi}{1+\sqrt{1-e^{2} \cos ^{2} \beta}} \\
& =-c e^{2} \int_{0}^{\psi} \frac{d \psi}{1+\sqrt{1-e^{2}} \sqrt{1+k^{2} \sin ^{2} \gamma(\psi)}}
\end{aligned}
$$

In the last step of Eq. (38d) we applied Corollary 1. It can be further refined to

$$
d \lambda=-\frac{c e^{2}}{2}\left\{\psi+e^{2} \int_{0}^{\psi} \frac{1-\left(1-c^{2}\right) \sin ^{2} \gamma(\psi) d \psi}{\left[1+\sqrt{1-e^{2}} \sqrt{1+k^{2} \sin ^{2} \gamma(\psi)}\right]^{2}}\right\}
$$

Here the integral can be determined by numerical integration. Alternatively, the integrand can be integrated similar to that for Eq. (34b).

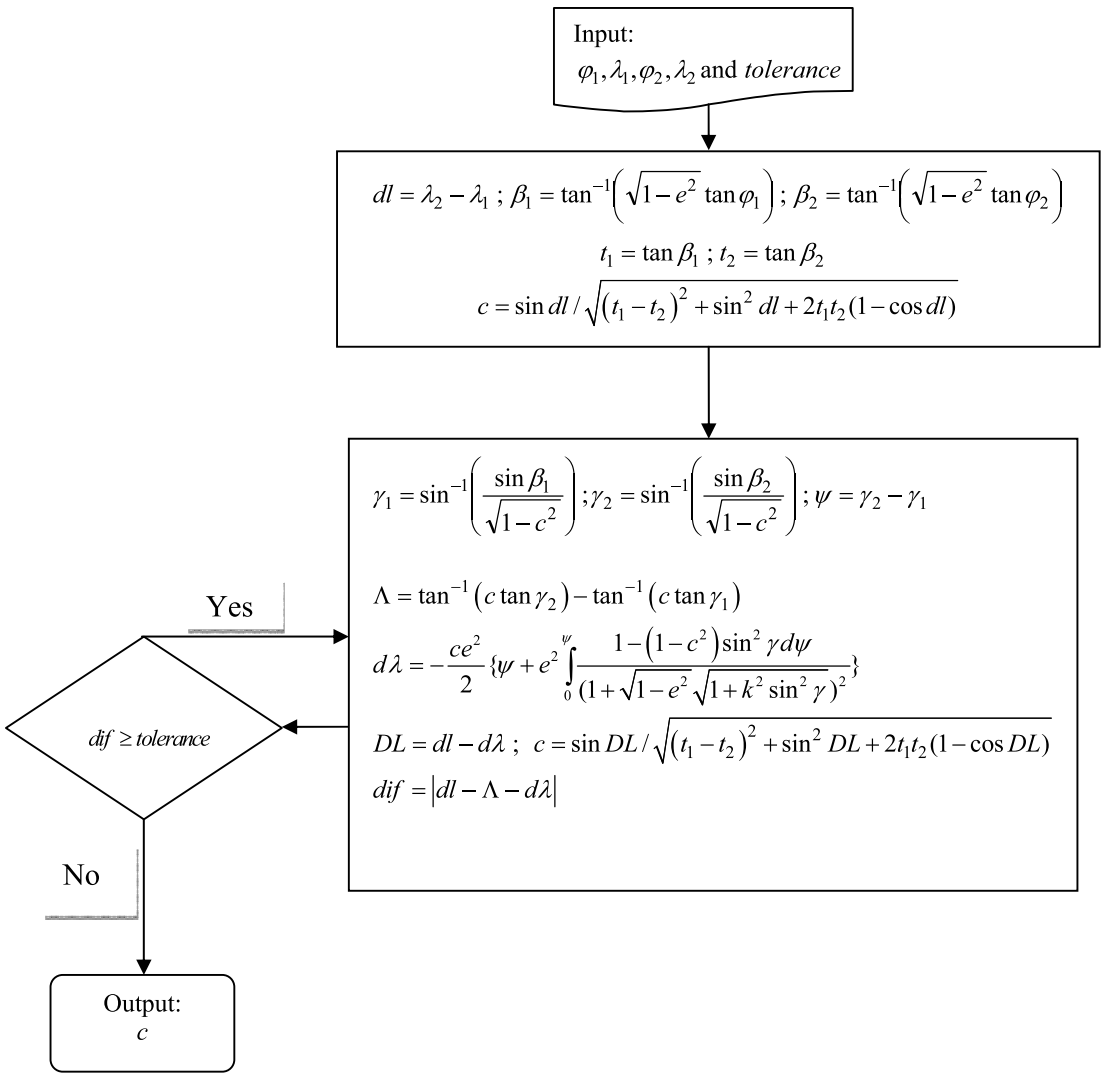

Fig. 2. Iteration scheme for solving $c$ (Clairaut's constant) 
A. Solving the direct problem.

Here $\beta_{1}, \lambda_{1}, s$, and $\alpha_{1}$ are known, while $\beta, \lambda$, and $\alpha$ at the end of the geodesic are the unknowns.

The solution is as follows:

- The Clairaut constant is determined by Eq. (3) applied at the given point;

- $\gamma_{1}$ follows from Eq. (31);

- By rewriting Eq. (34b) in the iterative form

$$
\begin{aligned}
\psi_{j+1}= & \frac{1}{1+k^{2} / 4}\left[\frac{s}{b}-\frac{k^{2}}{4} \frac{\sin 2 \gamma_{1}-\sin 2 \gamma_{j}}{2}\right. \\
& \left.+\frac{k^{4}}{2} \int_{0}^{\psi_{j}} \frac{\sin ^{4} \gamma}{\left(1+\sqrt{1+k^{2} \sin ^{2} \gamma}\right)^{2}} d \psi\right] \\
j= & 1,2, \ldots
\end{aligned}
$$

the iteration should converge well;

- $\beta$ follows from Proposition 1;

Table 1. Selected Points with Their Coordinates

\begin{tabular}{lcc}
\hline Station & $\begin{array}{c}\varphi \\
\left({ }^{\prime}{ }^{\prime \prime}\right)\end{array}$ & $\begin{array}{c}\lambda \\
\left({ }^{\prime}, \prime\right)\end{array}$ \\
\hline GRAZ & 470401.670268 & 0152936.534192 \\
PENC & 474722.573464 & 0191653.508828 \\
GANP & 490204.970940 & 0201922.573740 \\
ARTU & 562547.358444 & 0583337.651968 \\
NRIL & 692142.599412 & 0882135.220780 \\
BILI & 680434.063788 & 1662616.669464 \\
POINT2 & -380908.063980 & 1973242.463650 \\
\hline
\end{tabular}

Table 2. Discrepancies between Our Results and the Ones from Vincenty's Solution for the Inverse Geodetic Problem

\begin{tabular}{lccccccc}
\hline & \multicolumn{3}{c}{$\alpha$-formulas } & & \multicolumn{3}{c}{$\psi$-formulas } \\
\cline { 2 - 3 } Station & $\begin{array}{c}\Delta s \\
(\mathrm{~mm})\end{array}$ & $\begin{array}{c}\Delta A z_{1} \\
\left({ }^{\prime \prime}\right)\end{array}$ & $\begin{array}{c}\Delta A z_{2} \\
\left({ }^{\prime \prime}\right)\end{array}$ & & $\begin{array}{c}\Delta s \\
(\mathrm{~mm})\end{array}$ & $\begin{array}{c}\Delta A z_{1} \\
\left({ }^{\prime \prime}\right)\end{array}$ & $\begin{array}{c}\Delta A z_{2} \\
\left({ }^{\prime \prime}\right)\end{array}$ \\
\hline PENC & 0.0 & $3 \times 10^{-5}$ & $1 \times 10^{-5}$ & & 0.0 & $3 \times 10^{-5}$ & $1 \times 10^{-5}$ \\
GANP & 0.0 & $0 \times 10^{-5}$ & $2 \times 10^{-5}$ & & 0.0 & $0 \times 10^{-5}$ & $2 \times 10^{-5}$ \\
ARTU & 0.0 & $2 \times 10^{-5}$ & $1 \times 10^{-5}$ & & 0.0 & $2 \times 10^{-5}$ & $1 \times 10^{-5}$ \\
NRIL & 0.0 & $4 \times 10^{-5}$ & $4 \times 10^{-5}$ & & 0.0 & $4 \times 10^{-5}$ & $4 \times 10^{-5}$ \\
BILI & 0.0 & $2 \times 10^{-5}$ & $2 \times 10^{-5}$ & & 0.0 & $2 \times 10^{-5}$ & $2 \times 10^{-5}$ \\
POINT2 & - & - & - & & 3.1 & $1 \times 10^{-5}$ & $5 \times 10^{-5}$ \\
\hline
\end{tabular}

- $\lambda$ follows from Eqs. (38a), (38c), and (38e);

- $\alpha$ follows from Eq. (1) as follows: $\cos \alpha=\sqrt{\cos ^{2} \beta-c^{2}} /$ $\cos \beta$ or $\sin \alpha=c / \cos \beta$.

B. Solving the inverse problem.

We now assume that the coordinates of the terminal points are known, and it is requested to determine the length of the geodesic between the points and the azimuths of the geodesic at the points.

The solution is as follows:

- The Clairaut constant is determined as for the previously described inverse problem with Eqs. (24), (25a), and (25b), although $d \lambda$ can be more easily determined by Eq. (38d). For long arcs, $c$ must be computed with utmost precision;

- The azimuths at the terminal points are determined by Eq. (3);

- $\psi=\gamma-\gamma_{1}$; see Eqs. (27b) and (28);

- $s$ follows from Eq. (34b).

Computing $c$ with utmost precision can be achieved by using the following formulas:

$$
c=\sin D L / \sqrt{\left(t_{1}-t_{2}\right)^{2}+\sin ^{2} D L+2 t_{1} t_{2}(1-\cos D L)}
$$

where $D L$ was given by Eq. (25c), $d \lambda$ by Eq. (38e), and

$$
\psi=\gamma-\gamma_{1}=\arcsin \left(\frac{\sin \beta}{\sqrt{1-c^{2}}}\right)-\arcsin \left(\frac{\sin \beta_{1}}{\sqrt{1-c^{2}}}\right)
$$

Because $c$ is needed to determine $\psi$ and vice versa, the iteration scheme of Fig. 2 is recommended to be used to solve for both parameters.

\section{Numerical Tests}

To verify the accuracy of this new method and its consistency with other methods, we performed a test. Table 1 lists six International GPS and Geodynamics Services (IGS) stations plus POINT2, selected for the test. Station POINT2 was selected fairly close to the antipode of station GRAZ.

For the inverse geodetic problem, we computed the lengths and azimuths from GRAZ to the rest of the points. Table 2 shows the comparison between our results, according to the previously described procedures ( $\alpha$ - and $\psi$-formulas), and the results from Vincenty's method.

From Vincenty's solution for the inverse problem, we took lengths and forward azimuths to solve the direct problem using our method. The discrepancies between our results and the ones

\begin{tabular}{|c|c|c|c|c|c|c|c|c|}
\hline \multirow[b]{2}{*}{ Station } & \multirow[b]{2}{*}{$\begin{array}{c}s \\
(\mathrm{~m})\end{array}$} & \multirow[b]{2}{*}{$\begin{array}{c}A z_{1} \\
\left({ }^{\prime}{ }^{\prime} "\right)\end{array}$} & \multicolumn{3}{|c|}{$\psi$-Vincenty } & \multicolumn{3}{|c|}{$\alpha$-Vincenty } \\
\hline & & & $\begin{array}{l}\Delta \varphi \\
\left({ }^{\prime \prime}\right)\end{array}$ & $\begin{array}{c}\Delta \lambda \\
\left({ }^{\prime \prime}\right)\end{array}$ & $\begin{array}{c}\Delta A z_{2} \\
\left({ }^{\prime \prime}\right)\end{array}$ & $\begin{array}{c}\Delta \varphi \\
(")\end{array}$ & $\begin{array}{c}\Delta \lambda \\
(")\end{array}$ & $\begin{array}{c}\Delta A z_{2} \\
\left({ }^{\prime \prime}\right)\end{array}$ \\
\hline PENC & 0296830.8373 & 725443.5586 & $0 \times 10^{-5}$ & $1 \times 10^{-5}$ & $0 \times 10^{-5}$ & $1 \times 10^{-5}$ & $0 \times 10^{-5}$ & $0 \times 10^{-5}$ \\
\hline GANP & 0421181.2933 & 565606.0861 & $0 \times 10^{-5}$ & $1 \times 10^{-5}$ & $0 \times 10^{-5}$ & $2 \times 10^{-5}$ & $1 \times 10^{-5}$ & $3 \times 10^{-4}$ \\
\hline ARTU & 3091732.2259 & 541757.7978 & $1 \times 10^{-5}$ & $0 \times 10^{-5}$ & $0 \times 10^{-5}$ & $0 \times 10^{-5}$ & $3 \times 10^{-5}$ & $0 \times 10^{-5}$ \\
\hline NRIL & 4560739.5641 & 305956.1813 & $3 \times 10^{-5}$ & $1 \times 10^{-5}$ & $6 \times 10^{-5}$ & $3 \times 10^{-5}$ & $1 \times 10^{-5}$ & $1 \times 10^{-4}$ \\
\hline BILI & 7006861.3244 & 11467.5325 & $1 \times 10^{-5}$ & $5 \times 10^{-5}$ & $6 \times 10^{-5}$ & $1 \times 10^{-5}$ & $3.2 \times 10^{-3}$ & $1 \times 10^{-4}$ \\
\hline POINT2 & 19000000.0000 & 100000.0000 & $1 \times 10^{-4}$ & $2 \times 10^{-5}$ & $4 \times 10^{-5}$ & - & - & - \\
\hline
\end{tabular}
from Vincenty's formulas are shown in Table 3.

As shown in the tables, the results from the $\alpha$ - and $\psi$-formulas are consistent with one another. They are consistent with the results

Table 3. Direct Solution (from the Origin of GRAZ): Discrepancies between the Results from Our Methods ( $\alpha$ - and $\psi$-formulas) and Vincenty's Solution 
from Vincenty's method too. Although the results seem to be practically the same, the $\psi$-formulas show better behavior when dealing with longer baselines. For the last baseline, there is no result from the $\alpha$-formulas. This is because of the singularity in the formulas when crossing the equator.

\section{Concluding Remarks}

We have demonstrated how to solve the direct and inverse problems on the ellipsoid by adding the strict solution for the sphere and an ellipsoidal correction determined by numerical integration. By employing numerical integration, the routines which are usually available in current computer software like MATLAB (MATLAB function quadl), the problems of classical geodesy are easily solved to desired precision. Of particular concern for this study were the cases with singular integrands or nonunique solutions to integrals, i.e., when the integrals of the geodesic pass the vertices and/or the equator. To avoid such problems, the most practical solution is provided by Bessel's modified method, where the integrations take place along the great circle of an auxiliary sphere.

The tests show that both the direct and inverse problem solutions practically agree with Vincenty's solutions. Although Vincenty's iterative method apparently is more practical, our method, to our knowledge, is the only independent method for validating Vincenty's inverse method. It is also likely that the present method can be further improved.

\section{Acknowledgments}

We acknowledge the detailed comments by two unknown reviewers on a preliminary version of the manuscript.

\section{References}

Bessel, F. W. (1826). "Über die berechnung der geographischen längen und breiten aus geodätischen Vermessungen." Astron. Nachr., 4(86), 241-254.

Heck, B. (1987). Rechenverfahren und Auswertemodelle der Landesvermessung, Herbert Wichmann Verlag, Karlsruhe, Germany.

Kivioja, L. A. (1971). "Computation of geodetic direct and indirect problems by computers accumulating increments from geodetic line elements." Bull. Geod., 99(1), 55-63.

Klotz, J. (1991). "Eine analytische Lösung kanonischer Gleichungen der geodätischen Linien zur Transformation ellipsoidischer Flächenkoordinaten." Deutsche Geod. Kommission Ser. C, No. 385, Bavarian Academy of Sciences, Munich.

Rainsford, H. F. (1955). "Long geodesics on the ellipsoid." Bull. Geod., $37(1), 12-22$.

Schmidt, H. (1999). "Lösung der geodätischen Hauptaufgaben auf dem Rotationsellipsoid mittels numerischer Integration." Z. Vermessungsw. 124, 121-128.

Schmidt, H. (2006). "Note on Lars E. Sjöberg: New solutions to the direct and indirect geodetic problems on the ellipsoid." Z. Vermessungsw., 131, 153-154.

Sjöberg, L. E. (2006a). "New solutions to the direct and indirect geodetic problems on the ellipsoid." Zeitschrift fuer Vermessungswesen, 131, 35-39.

Sjöberg, L. E. (2006b). "Comment to H. Schmidt's remarks on Sjöberg, zfv 1/2006, 35-39.” Z. Vermessungsw., 131, 155.

Sjöberg, L. E. (2006c). "Determination of areas on the plane, sphere and ellipsoid.” Surv. Rev., 38, 583-593.

Sjöberg, L. E. (2007). "Precise determination of the Clairaut constant in ellipsoidal geodesy." Surv. Rev., 39(303), 81-86.

Thomas, C. M., and Featherstone, W. E. (2005). "Validation of Vincenty's formulas for the geodesic using a new fourth-order extension of Kivioja's formula." J. Surv. Eng., 131(1), 20-26.

Vincenty, T. (1975). "Direct and inverse solutions of geodesics on the ellipsoid with application of nested equations." Surv.Rev., 23(176), 88-93. 